Review began 12/16/2021 Review ended 01/18/2022 Published 01/25/2022

๑) Copyright 2022

Amano et al. This is an open access article distributed under the terms of the Creative Commons Attribution License CC-BY 4.0., which permits unrestricted use, distribution, and reproduction in any medium, provided the original author and source are credited.

\section{Preoperative Prediction of Intracranial Meningioma Grade Using Conventional CT and MRI}

Toshiyuki Amano ${ }^{1}$, Akira Nakamizo ${ }^{1}$, Hideki Murata ${ }^{1}$, Yuichiro Miyamatsu ${ }^{1}$, Fumihito Mugita ${ }^{1}$, Koji Yamashita $^{2}$, Tomoyuki Noguchi ${ }^{2}$, Shinji Nagata ${ }^{1}$

1. Neurosurgery, Kyushu Medical Center, Fukuoka, JPN 2. Radiology, Kyushu Medical Center, Fukuoka, JPN

Corresponding author: Toshiyuki Amano, amano@ns.med.kyushu-u.ac.jp

\section{Abstract \\ Objective}

Preoperative diagnosis of tumor grade can assist in treatment-related decision-making for patients with intracranial meningioma. This study aimed to distinguish between high-grade and low-grade meningiomas using conventional CT and MRI.

\section{Methodology}

We retrospectively analyzed 173 consecutive patients with intracranial meningioma (149 low-grade and 24 high-grade tumors) who were treated surgically at the National Hospital Organization Kyushu Medical Center from 2008 to 2020. Clinical and radiological features, including tumor doubling time (Td) and relative growth rate (RGR), were compared between low-grade and high-grade meningiomas.

\section{Results}

Multivariate logistic regression analysis showed that symptomatic tumor ( $\mathrm{p}=0.001)$, non-skull base location $(\mathrm{p}=0.006)$, irregular tumor shape $(\mathrm{p}=0.043)$, tumor heterogeneity $(\mathrm{p}=0.025)$, and peritumoral brain edema $(\mathrm{p}=0.003)$ were independent predictors of high-grade meningioma. In 53 patients who underwent surgery because of tumor progression, progression to symptoms $(\mathrm{p}=0.027)$, intratumoral heterogeneity $(\mathrm{p}<0.001)$, peritumoral brain edema ( $\mathrm{p}=0.001)$, larger tumor volume $(\mathrm{p}=0.005)$, shorter $\mathrm{Td}(\mathrm{p}<0.001)$, and higher RGR $(\mathrm{P}<0.001)$ were significantly associated with high-grade meningioma. Receiver operating characteristics (ROC) curve analysis showed that the optimal Td and annual RGR cut-off values to distinguish high-grade from low-grade meningioma were 460.5 days and $73.2 \%$, respectively (100\% sensitivity and $78.6 \%$ specificity).

\section{Conclusion}

Based on our findings, conventional CT and MRI are useful methods to predict meningioma grades before surgery. High-grade lesions are associated with non-skull base location, irregular tumor shape, intratumoral heterogeneity, and peritumoral brain edema. High-grade meningioma should be suspected in tumors that exhibit $\mathrm{Td}<460.5$ days or annual RGR $>73.2 \%$ or those that develop intratumoral heterogeneity or surrounding brain edema on surveillance imaging.

Categories: Radiology, Neurosurgery

Keywords: relative growth rate, tumor doubling time, chronological changes, radiological features, preoperative diagnosis, intracranial high-grade meningioma

\section{Introduction}

Intracranial meningioma is the most common primary brain tumor. These tumors typically have an indolent course due to their benign biological features. However, high-grade meningiomas have been associated with faster progression, higher recurrence, and lower survival despite multimodal treatment [1]. The World Health Organization (WHO) tumor grade is an effective predictor of tumor recurrence and overall survival. This classification system for brain tumors was first introduced in 1979 and most recently updated in 2016. Although several recent systematic reviews and meta-analyses have explored possible predictors of the WHO meningioma grade, high-quality evidence is still scarce [2-5]. Recent advances in novel imaging techniques such as machine learning-based radiomics analysis can improve the accuracy of radiological prediction of meningioma grade [6,7]. However, most imaging markers remain insufficient for routine clinical use because of poor tumor grade prediction accuracy [3]. Therefore, preoperative diagnosis of highgrade meningiomas remains a challenge.

The 2016 WHO classification incorporated cerebral invasion into the diagnostic criteria for WHO grade II 
meningioma. This modification has resulted in an increase in high-grade meningioma diagnosis, which is estimated to range between $17 \%$ and $31 \%[8,9]$; based on the old WHO classification, the reported prevalence rates ranged from $5 \%$ to $10 \%$ [10]. Considering the cerebral invasion modification, radiological features of conventional CT and MRI may now be able to better predict meningioma grades.

In this study, we retrospectively analyzed 173 consecutive patients with histopathologically proven intracranial meningioma who underwent surgical resection at a single center. Clinical and radiological features were examined and compared between high-grade and low-grade tumors to assess the value of using preoperative CT and MRI to predict histopathological tumor grade.

\section{Materials And Methods}

This study was approved by the National Hospital Organization Kyushu Medical Center Research Ethics Board (\#17C292). Informed consent was obtained from all patients. In our hospital, asymptomatic small intracranial meningiomas are treated conservatively and observed using repeated radiological assessment. When these tumors demonstrate chronological progression, surgical removal is considered. However, early surgical intervention is considered for some asymptomatic tumors based on their size or location before observing tumor growth. Decisions regarding surgical indications and the timing of surgery are made by the attending neurosurgeon.

\section{Patient characteristics}

This retrospective study examined the medical and radiological records of consecutive patients with histopathologically confirmed meningioma at the National Hospital Organization Kyushu Medical Center from 2008 to 2020. Patients with neurofibromatosis, spinal meningioma, or recurrent meningioma were excluded. A total of 173 patients were included for analysis (129 females and 44 males). The median age of the cohort was 65 years (range: 30-96). Age, gender, comorbidities, symptoms, tumor location, and histopathological grade were recorded. The histopathological grade was determined according to the 2016 WHO criteria. WHO grade I meningiomas were categorized as low-grade, and grade II or III meningiomas as high-grade. Presenting symptoms included cranial nerve palsy, motor weakness, memory disturbance, seizure, headache, ataxia, gait disturbance, and dysesthesia. Tumor location was categorized as skull base (including the olfactory groove, frontal base, tuberculum sellae, clinoidal region, sphenoid ridge, middle fossa, clivus, petrous ridge, tentorium cerebelli, jugular foramen, and foramen magnum) or non-skull base (including the convexity, parasagittal angle, falx cerebri, and within the ventricle).

\section{Radiological findings}

All intracranial meningiomas were confirmed by CT and MRI. Radiological images were interpreted by a senior neuroradiologist experienced in neuroimaging who was blinded to the histopathological results. Bone erosion, hyperostosis of the adjacent skull, and tumor calcification were assessed on CT. MRI was performed using a $1.5 \mathrm{~T}$ or $3 \mathrm{~T}$ scanner to obtain T1-weighted imaging (T1-WI), T2-weighted imaging (T2-WI), fluidattenuated inversion recovery (FLAIR) imaging, and contrast-enhanced T1-WI. Tumor shape, intratumoral enhancement, and peritumoral brain edema were assessed on MRI. The irregular shape was defined as a lobulated (Figure $1 A$ ) or mushrooming appearance (Figure 1B). Intratumoral heterogeneity was defined as a heterogeneous enhancement on contrast-enhanced T1-WI (Figures $1 C, 1 D$ ). Peritumoral brain edema was defined as a hyperintense signal change in the adjacent brain parenchyma on FLAIR or T2-WI (Figures 1E, $1 F)$. Tumor size was measured as the maximum diameter on contrast-enhanced T1-WI. Tumor volume was calculated using the ellipsoid formula: (maximum length $\times$ maximum width $\times$ maximum height) $/ 2[2,11]$. When serial clinical and imaging data were available, additional factors such as progressive symptoms, initial tumor volume, final tumor volume, time from diagnosis to surgery, tumor doubling time (Td), and relative growth rate (RGR) were also examined. Td was calculated using the following formula: $\mathrm{T} \times \log 2 / \log$ $\left(\mathrm{V}_{1} / \mathrm{V}_{0}\right)$, where $\mathrm{T}$ is the time between the initial and final volume, $\mathrm{V}_{0}$ is the initial tumor volume, and $\mathrm{V}_{1}$ is the final tumor volume $[4,12]$. RGR was calculated using the following formula: $\left(2^{(365 / T d)}-1\right) \times 100$ [4]. 


\section{Cureus}
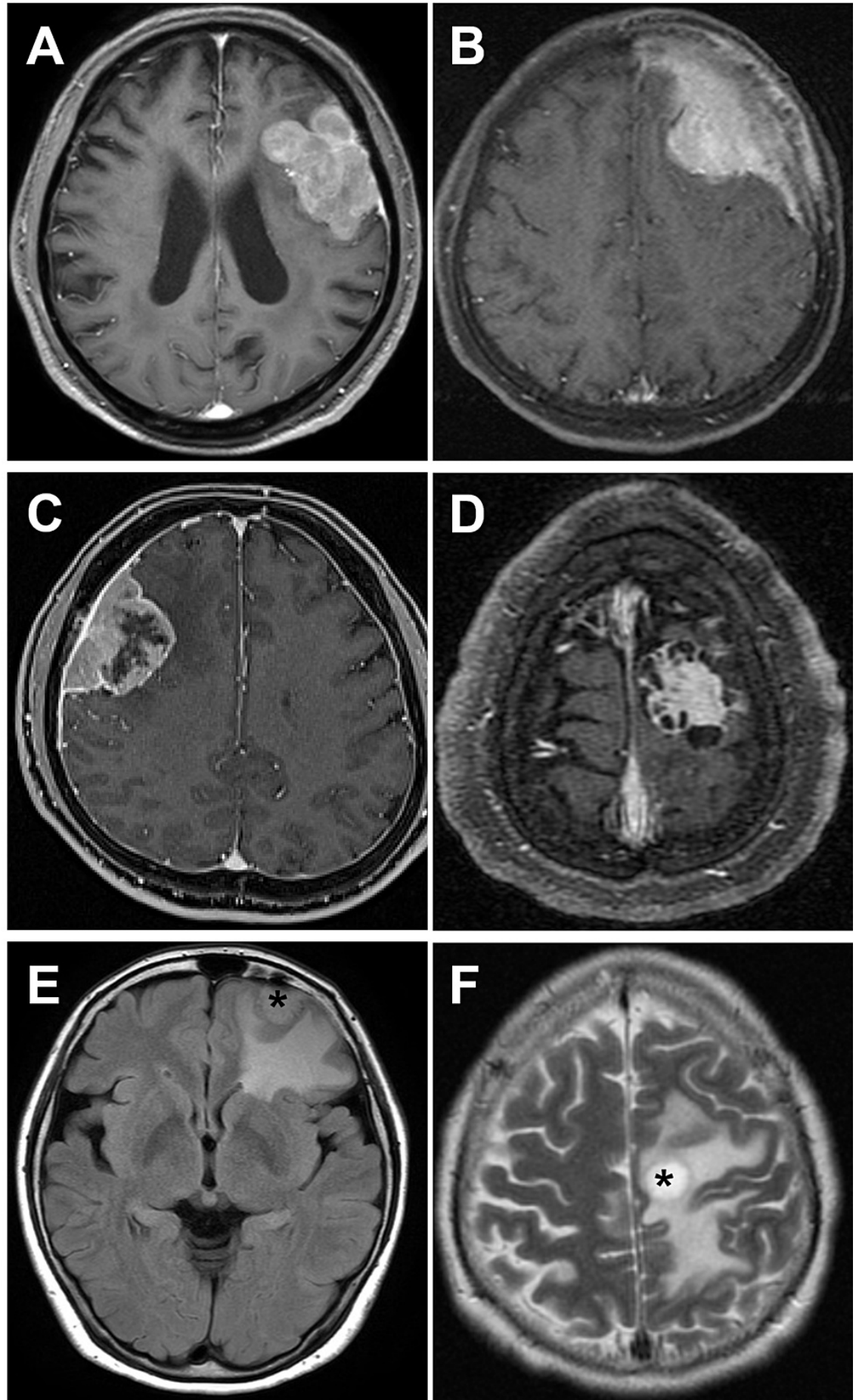

\section{FIGURE 1: Representative MRI}

MRI showing representative axial images of irregular tumor shape, such as lobulated appearance $(A)$ or mushrooming appearance $(B)$, and heterogeneous tumor enhancement $(C$ and $D)$ on contrast-enhanced T1weighted imaging and peritumoral brain edema on fluid-attenuated inversion recovery imaging $(\mathrm{E})$ and $\mathrm{T} 2$ weighted imaging $(F)$. The asterisk indicates tumor location

MRI: magnetic resonance imaging

\section{Statistical analyses}

Statistical analyses were performed using the R software version 4.0.0 (R Foundation for Statistical Computing, Vienna, Austria) [13]. The chi-square test was used to compare categorical variables between the low-grade and high-grade groups; age, tumor volume, interval to surgery, Td, and RGR were compared using the Wilcoxon test. Variables significantly associated with high-grade meningioma in univariate analysis 


\section{Cureus}

were used in multivariate logistic regression to assess independent predictors. The results of logistic regression are presented as odds ratios (ORs) with 95\% confidence intervals ( $95 \% \mathrm{CI}$ ). The feasibility of $\mathrm{Td}$ and RGR as predictors of high-grade meningioma was determined using receiver operating characteristic (ROC) curve analysis. The point lying closest to the upper left corner of the ROC curve was defined as the optimal cutoff threshold value. A p-value $<0.05$ was considered statistically significant.

\section{Results}

\section{Clinical and radiological features associated with high-grade meningioma}

Among the 173 meningioma patients, 149 (86.1\%) harbored a low-grade tumor and 24 (13.9\%) had a highgrade tumor. All high-grade tumors were WHO grade II. Patient characteristics are presented in Table 1. High-grade meningioma was significantly associated with older age (74 vs. 64 years; $\mathrm{p}=0.031$ ), symptomatic tumor (79.2\% vs. 38.3\%; $\mathrm{p}<0.001$ ), and non-skull base location (75.0\% vs. $47.7 \%$; $\mathrm{p}=0.023)$. However, gender $(\mathrm{p}=0.481)$, comorbidities (all $\mathrm{p}>0.1$ ), and timing of surgical intervention $(\mathrm{p}=0.684)$ did not differ between the groups. In multivariate logistic regression analysis, symptomatic tumor (OR: 6.49; 95\% CI: 2.33-21.34;

$\mathrm{p}=0.001$ ) and non-skull base location (OR: 4.39; 95\% CI: 1.62-13.57; $\mathrm{p}=0.006$ ) were independently associated with high-grade meningioma (Table 2).

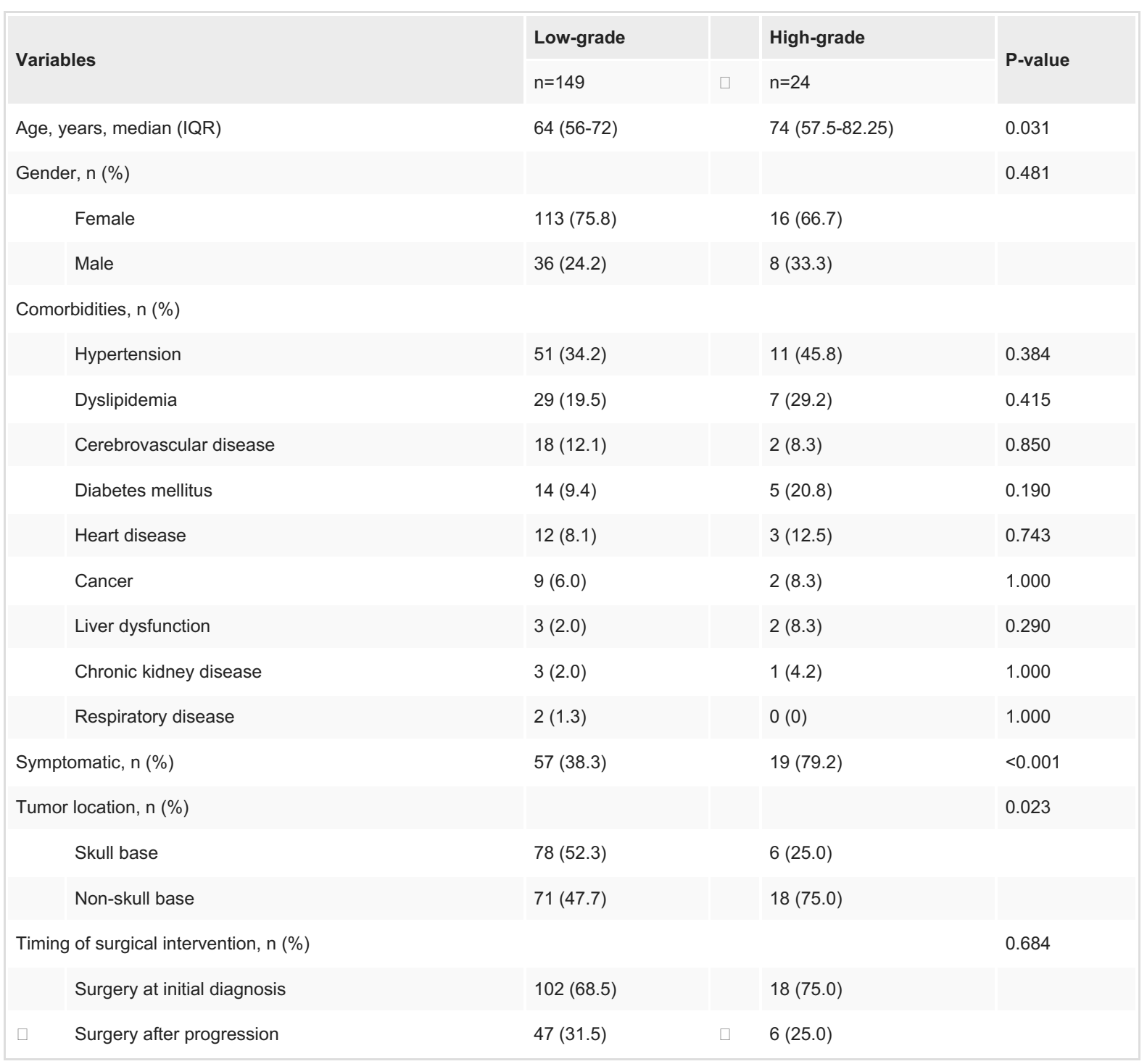

\section{TABLE 1: Clinical characteristics of 173 patients with intracranial meningioma}

IQR: interquartile range 


\section{Cureus}

\begin{tabular}{|l|lll|}
\hline Variables & \multicolumn{2}{l|}{ Multivariate analysis } & \\
\hline Age & OR & $95 \% \mathrm{Cl}$ & P-value \\
Symptomatic tumor & 1.04 & $1.00-1.08$ & 0.074 \\
Non-skull base tumor & 6.49 & $2.33-21.34$ & 0.001 \\
\hline
\end{tabular}

TABLE 2: Multivariate logistic regression analysis of associations between variables and highgrade meningioma in 173 patients

OR: odds ratio; $\mathrm{Cl}$ : confidence interval

Radiological findings are presented in Table 3. Bone erosion or hyperostosis in the adjacent skull was present in 139 patients (34 patients harbored tumors of the falx cerebri, tentorium cerebelli, or within the ventricle that were not adjacent to the skull). There were no significant differences in terms of hyperostosis $(\mathrm{p}=0.214)$ or calcification $(\mathrm{p}=0.155)$ between the low-grade and high-grade meningioma groups. Frequencies of bone erosion in the adjacent skull (35.0\% vs. $10.9 \%$; $\mathrm{p}=0.013)$, irregular tumor shape (45.8\% vs. $7.4 \%$; $\mathrm{p}<0.001)$, tumor heterogeneity (41.7\% vs. $5.4 \%$; $<<0.001)$, and peritumoral edema ( $91.7 \%$ vs. $34.2 \%$; $<<0.001)$ were significantly higher in the high-grade meningioma group. Preoperative tumor volume was significantly higher in the high-grade meningioma group $\left(30.6 \mathrm{~cm}^{3}\right.$ vs. $\left.8.3 \mathrm{~cm}^{3} ; \mathrm{p}<0.001\right)$. In multivariate logistic regression analysis (Table 4 ), the following variables were independently associated with high-grade meningioma: irregular tumor shape (OR: 4.04; 95\% CI: 1.04-16.11; $\mathrm{p}=0.043$ ), intratumoral heterogeneity (OR: 5.62; 95\% CI: 1.28-27.57, $\mathrm{p}=0.025$ ), and peritumoral brain edema (OR: 13.35; 95\% CI: 2.85-99.35; $\mathrm{p}=0.003)$.

\begin{tabular}{|c|c|c|c|c|}
\hline \multirow{2}{*}{ Variables } & Low-grade & $\square$ & High-grade & \multirow{2}{*}{ P-value } \\
\hline & $n=149$ & $\square$ & $n=24$ & \\
\hline Bone erosion, n (\%) & 13/119 (10.9) & & 7/20 (35.0) & 0.013 \\
\hline Hyperostosis, n (\%) & 14/119 (11.8) & & $5 / 20(25.0)$ & 0.214 \\
\hline Calcification, n (\%) & $57 / 149(38.3)$ & & 5/24 (20.8) & 0.155 \\
\hline Irregular tumor shape, n (\%) & 11/149 (7.4) & & $11 / 24(45.8)$ & $<0.001$ \\
\hline Intratumoral heterogeneity, n (\%) & 8/149 (5.4) & & $10 / 24(41.7)$ & $<0.001$ \\
\hline Peritumoral brain edema, n (\%) & $51 / 149(34.2)$ & & 22/24 (91.7) & $<0.001$ \\
\hline Tumor volume, $\mathrm{cm}^{3}$, median (IQR) & $8.3(3.7-19.7)$ & $\square$ & $30.6(16.7-50.8)$ & $<0.001$ \\
\hline
\end{tabular}

TABLE 3: Radiological findings of $\mathbf{1 7 3}$ patients with intracranial meningioma

IQR: interquartile range 


\section{Cureus}

\begin{tabular}{|c|c|c|c|}
\hline \multirow{2}{*}{ Variables } & \multicolumn{3}{|c|}{ Multivariate analysis } \\
\hline & OR & $95 \% \mathrm{Cl}$ & P-value \\
\hline Bone erosion & 1.92 & $0.42-8.29$ & 0.383 \\
\hline Irregular tumor shape & 4.04 & $1.04-16.11$ & 0.043 \\
\hline Intratumoral heterogeneity & 5.62 & $1.28-27.57$ & 0.025 \\
\hline Peritumoral brain edema & 13.35 & $2.85-99.35$ & 0.003 \\
\hline Tumor volume & 0.99 & $0.97-1.01$ & 0.306 \\
\hline
\end{tabular}

TABLE 4: Multivariate logistic regression analysis of associations between variables and highgrade meningioma in 173 patients

OR: odds ratio; $\mathrm{Cl}$ : confidence interval

\section{Chronological changes associated with high-grade meningioma}

As shown in Table 1, 53 patients (47 with low-grade and six with high-grade meningioma) underwent a surgical intervention because of tumor progression. Among these patients, we investigated chronological changes in clinical and radiological characteristics, which are shown in Table 5. High-grade meningioma was significantly associated with progression to symptoms ( $66.7 \%$ vs. $17.0 \%$; $\mathrm{p}=0.027$ ), acquisition of intratumoral heterogeneity ( $66.7 \%$ vs. $2.1 \%$; $p<0.001)$ and peritumoral brain edema $(100 \%$ vs. $23.4 \%$; $\mathrm{p}=0.001)$, larger final tumor volume $\left(28.7 \mathrm{~cm}^{3}\right.$ vs. $\left.5.9 \mathrm{~cm}^{3} ; \mathrm{p}=0.005\right)$, shorter $\mathrm{Td}$ (304 days vs. 1038 days; $\mathrm{p}<0.001)$, and higher RGR (133\% vs. $28 \%$; $\mathrm{p}<0.001)$. However, there were no differences in terms of age $(\mathrm{p}=0.066)$, gender $(\mathrm{p}=0.549)$, acquisition of irregular tumor shape $(\mathrm{p}=0.534)$, initial tumor volume $(\mathrm{p}=0.066)$, and time from diagnosis to surgery $(\mathrm{p}=0.218)$ between the groups. ROC curve analysis showed that Td could distinguish between high- and low-grade meningiomas with a sensitivity of $100 \%$ and specificity of $78.6 \%$ using a cut-off of 460.5 days [area under the curve (AUC): 0.90; Figure 2]. RGR could distinguish between the two with a sensitivity of $100 \%$ and specificity of $78.6 \%$ using a cut-off of $73.2 \%$ per year (AUC: 0.90 ; Figure 3). 


\section{Cureus}

\begin{tabular}{|c|c|c|c|c|c|}
\hline \multirow{2}{*}{ Variables } & Low-grade & & High-grade & & \multirow{2}{*}{ P-value } \\
\hline & $\mathrm{n}=47$ & $\square$ & $\mathrm{n}=6$ & $\square$ & \\
\hline Age, years, median (IQR) & $68(60-73)$ & & $82(78.75-83.75)$ & & 0.066 \\
\hline Gender, n (\%) & & & & & 0.549 \\
\hline Female & $38(80.9)$ & & $6(100)$ & & \\
\hline Male & $9(19.1)$ & & $0(0)$ & & \\
\hline Progression to symptoms, $\mathrm{n}(\%)$ & $8(17.0)$ & & $4(66.7)$ & & 0.027 \\
\hline \multicolumn{6}{|l|}{ Acquired radiological features, $\mathrm{n}(\%)$} \\
\hline Irregular tumor shape & $1(2.1)$ & & $1(16.7)$ & & 0.534 \\
\hline Intratumoral heterogeneity & $1(2.1)$ & & $4(66.7)$ & & $<0.001$ \\
\hline Peritumoral brain edema & $11(23.4)$ & & $6(100)$ & & 0.001 \\
\hline Initial tumor volume, $\mathrm{cm}^{3}$, median (IQR) & $1.5(0.7-3.4)$ & & $4.0(2.9-7.3)$ & & 0.066 \\
\hline Final tumor volume, $\mathrm{cm}^{3}$, median (IQR) & $5.9(2.6-9.8)$ & & $28.7(19.8-32.0)$ & & 0.005 \\
\hline Time from diagnosis to surgery, days, median (IQR) & $1060(625-1605)$ & & $638(427-977)$ & & 0.218 \\
\hline Tumor doubling time, days, median (IQR) & 1038 (504-1614) & & $304(174-424)$ & & $<0.001$ \\
\hline Relative growth rate, \%, median (IQR) & $28(17-65)$ & $\square$ & $133(84-415)$ & $\square$ & $<0.001$ \\
\hline
\end{tabular}

TABLE 5: Clinical and radiological features of 53 patients with progressive intracranial meningioma

IQR: interquartile range 


\section{Cureus}

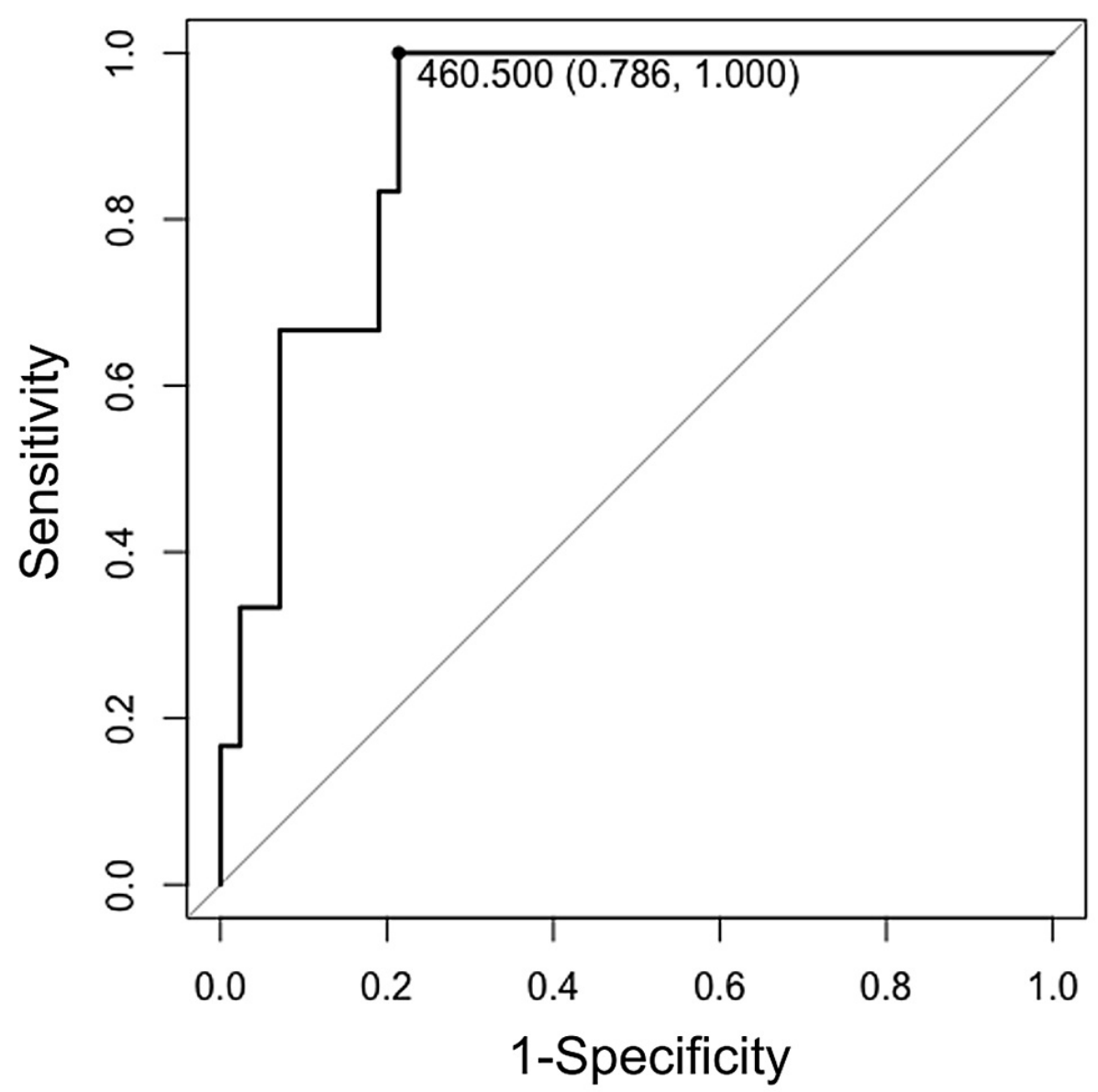

FIGURE 2: The receiver operating characteristic (ROC) curve of tumor doubling time ( $\mathrm{Td})$ in $\mathbf{5 3}$ progressive tumors

The receiver operating characteristics (ROC) curve of tumor doubling time (Td) in progressive tumors to differentiate between high-grade and low-grade meningioma. The area under the curve was 0.90 . The optimal cutoff value was 460.5 days. The specificity and sensitivity were $78.6 \%$ and $100 \%$, respectively 


\section{Cureus}

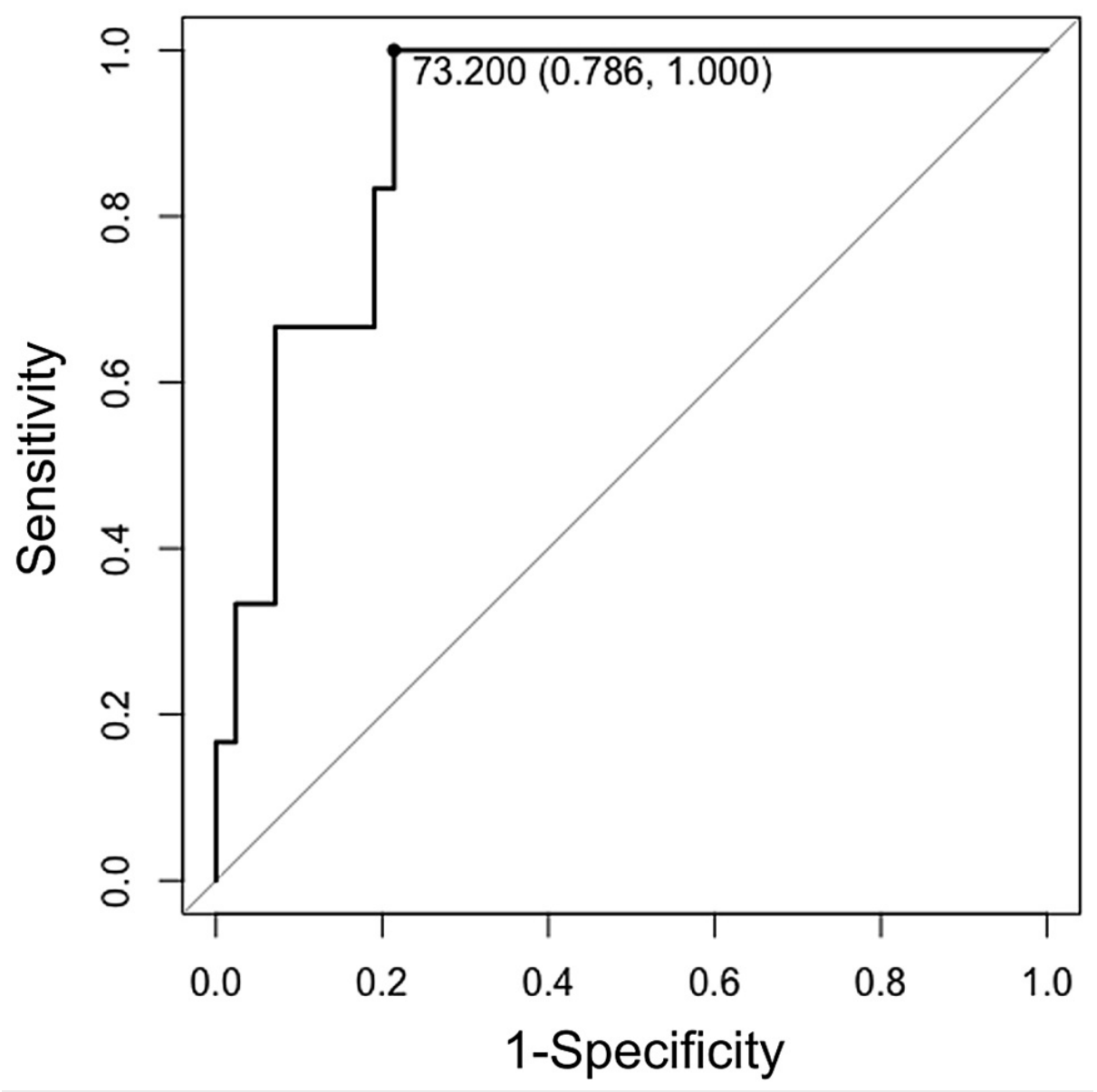

FIGURE 3: The receiver operating characteristic (ROC) curve of relative growth rate (RGR) in $\mathbf{5 3}$ progressive tumors

The receiver operating characteristics (ROC) curve of relative growth rate (RGR) in progressive tumors to differentiate between high-grade and low-grade meningioma. The area under the curve was 0.90 . The optimal cutoff value was $73.2 \%$ per year. The specificity and sensitivity were $78.6 \%$ and $100 \%$, respectively

Discussion

\section{Clinical and radiological features associated with high-grade meningioma}

In the present study, we attempted to determine whether preoperative CT and MRI can distinguish WHO high-grade intracranial meningioma from the low-grade variant. Although previous studies have also explored possible predictors of meningioma grade or subtype based on clinical characteristics or radiological features, the results have been conflicting. For example, the presence of peritumoral brain edema has been reported as a predictor of higher tumor grade in some studies [1,14] but not in others $[15,16]$. Therefore, preoperative diagnosis of high-grade meningiomas remains both controversial and challenging, and hence further data and analysis are still needed. In agreement with other studies, our research showed that symptomatic tumor [17], non-skull base location [14,17], irregular tumor shape [1,14,16], intratumoral heterogeneity $[1,6,16]$, and the presence of peritumoral brain edema appear to be independent predictors of high-grade meningioma. The fact that clinical and radiologic findings can change over time must be considered when evaluating meningioma patients. The distinctive radiological features mentioned above may not be detected in newly arising meningiomas, even high-grade ones; rather, they may be gradually acquired as the tumor progresses. Therefore, although these radiological features can be associated with high-grade meningioma, their absence does not always indicate that the tumor is benign. Taken together, earlier surgical intervention with more radical resection should be considered in cases of non-skull base location, irregular tumor shape, intratumoral heterogeneity, and peritumoral brain edema, even in patients with an incidental tumor.

In contrast with previous studies, older age, male gender, comorbidities, bone erosion, absence of calcification, and tumor volume were not independently associated with high-grade meningioma in our study [7,16-18]. These conflicting results are likely due to variations in study design and population between 
studies. However, all studies, including ours, were conducted among patients with histopathologically confirmed meningioma; patients with an apparent meningioma followed conservatively or treated with radiosurgery without biopsy were excluded. In patients with an asymptomatic small tumor, conservative treatment or radiotherapy is generally preferred for older patients in poor condition, while surgical intervention may be preferred for younger healthy patients. Treatment decision-making for asymptomatic meningiomas is based on patient characteristics such as age, symptoms, and comorbidities as well as tumor characteristics such as location and size; patient preference may also be a factor [19]. Sample selection bias may have been present in several studies, making comparison difficult. To address this issue, standardized criteria should be established and used in future studies.

\section{Chronological changes in progressive meningioma}

With the widespread use of CT and MRI and advances in imaging techniques, incidental asymptomatic intracranial meningiomas are frequently detected these days. Nakasu and Nakasu, in a systematic review and meta-analysis of meningioma natural history, have shown that approximately $30 \%$ of incidental meningioma do not grow further [4]. When a meningioma has no distinct radiological high-grade features, conservative management is a reasonable option for asymptomatic small tumors. However, approximately $70 \%$ of intracranial meningiomas show chronological progression. Earlier establishment of tumor grade in progressive tumors can assist in treatment decision-making. When serial imaging data are available, chronological changes in tumor characteristics can be analyzed. We explored potential factors that distinguish between high- and low-grade lesions in progressive meningiomas. Some progressive meningiomas did not initially have the distinctive clinical and radiological features mentioned above but acquired them during observation. Among these, progression to symptoms, intratumoral heterogeneity, and peritumoral brain edema were significantly associated with high-grade meningioma; however, irregular tumor shape was not. This suggests that intratumoral heterogeneity and peritumoral brain edema are likely to be present in progressive high-grade meningioma. Development of these changes on imaging should be a focus of observation and raise suspicion for a high-grade tumor when identified.

Larger final tumor volume, shorter Td, and higher RGR were significantly associated with high-grade meningioma in our study. Although previous studies have also suggested an association between shorter Td and high-grade tumors [20-22], these studies used the old WHO meningioma classification, were limited by small sample size, did not conduct statistical analyses, and some of them included patients with unknown histology. To our knowledge, our study is the first to demonstrate that Td is significantly associated with meningioma grade and can be a reliable predictor of high-grade meningioma. Our ROC curve analysis showed a high AUC value (0.90) for both Td and RGR, indicating excellent performance at distinguishing high-grade from low-grade. The optimal Td cut-off value was 460.5 days, suggesting that tumor volume doubling within 15.2 months likely indicates high-grade meningioma. The optimal RGR cut-off value was $73.2 \%$ per year. This growth is equivalent to an estimated $20 \%$ increase in maximum length, width, and height. Such growth measured in one year on radiological surveillance imaging probably indicates highgrade meningioma. Taken together, Td and RGR can be possible predictors of high-grade meningioma even in tumors without other concerning radiological findings.

\section{Limitations}

This study has several limitations. Firstly, the retrospective single-center design may have introduced biases that affect the generalizability of our findings. For example, the surgical indications and timing in each patient were dependent on the individual treating neurosurgeon, and selection bias may have been present. Hence, future studies using established standardized surgical criteria are required. Secondly, the sample size was relatively small, which may have contributed to the lack of significance in some analyses. Because de novo intracranial high-grade meningioma is relatively rare, future multicenter studies are necessary to confirm our findings.

\section{Conclusions}

In the present study, we attempted to correlate clinical and radiological features of intracranial meningioma with histopathological grading according to the revised 2016 WHO classification. Based on our findings, symptomatic tumor, non-skull base location, irregular tumor shape, intratumoral heterogeneity, and peritumoral brain edema were independent predictors of high-grade meningioma. When these distinctive clinical and radiological features are observed in patients with suspected meningioma, earlier surgical intervention with more radical resection should be considered.

In progressive meningiomas, those that developed intratumoral heterogeneity and peritumoral brain edema were likely to be high-grade. Furthermore, shorter Td and higher RGR were significantly associated with tumor grade, suggesting that these parameters may be predictors of high-grade meningioma. When evaluating chronological changes in meningioma, the presence of intratumoral heterogeneity, peritumoral brain edema, $\mathrm{Td}<460.5$ days, and annual RGR $>73.2 \%$ should raise suspicion for a high-grade tumor.

\section{Additional Information}




\section{Disclosures}

Human subjects: Consent was obtained or waived by all participants in this study. National Hospital Organization Kyushu Medical Center Research Ethics Board issued approval 17C292. This study has been approved by the National Hospital Organization Kyushu Medical Center Research Ethics Board. Animal subjects: All authors have confirmed that this study did not involve animal subjects or tissue. Conflicts of interest: In compliance with the ICMJE uniform disclosure form, all authors declare the following: Payment/services info: All authors have declared that no financial support was received from any organization for the submitted work. Financial relationships: All authors have declared that they have no financial relationships at present or within the previous three years with any organizations that might have an interest in the submitted work. Other relationships: All authors have declared that there are no other relationships or activities that could appear to have influenced the submitted work.

\section{Acknowledgements}

We thank the members of the pathology team (Drs. S. Momosaki, S. Kawauchi, and M. Fujiwara) at the National Hospital Organization Kyushu Medical Center for their contributions, and Edanz (https://jp.edanz.com/ac) for editing a draft of this manuscript.

\section{References}

1. Yu J, Chen FF, Zhang HW, et al.: Comparative analysis of the MRI characteristics of meningiomas according to the 2016 WHO pathological classification. Technol Cancer Res Treat. 2020, 19:1533033820983287. $10.1177 / 1533033820983287$

2. Fountain DM, Soon WC, Matys T, Guilfoyle MR, Kirollos R, Santarius T: Volumetric growth rates of meningioma and its correlation with histological diagnosis and clinical outcome: a systematic review. Acta Neurochir (Wien). 2017, 159:435-45. 10.1007/s00701-016-3071-2

3. Huang RY, Bi WL, Griffith B, et al.: Imaging and diagnostic advances for intracranial meningiomas . Neuro Oncol. 2019, 21:i44-61. 10.1093/neuonc/noy143

4. Nakasu S, Nakasu Y: Natural history of meningiomas: review with meta-analyses . Neurol Med Chir (Tokyo). 2020, 60:109-20. 10.2176/nmc.ra.2019-0213

5. Siempis T, Tsakiris C, Alexiou GA, Xydis VG, Voulgaris S, Argyropoulou MI: Diagnostic performance of diffusion and perfusion MRI in differentiating high from low-grade meningiomas: a systematic review and meta-analysis. Clin Neurol Neurosurg. 2020, 190:105643. 10.1016/j.clineuro.2019.105643

6. Coroller TP, Bi WL, Huynh E, et al.: Radiographic prediction of meningioma grade by semantic and radiomic features. PLoS One. 2017, 12:e0187908. 10.1371/journal.pone.0187908

7. $\mathrm{Hu}$ J, Zhao Y, Li M, et al.: Machine learning-based radiomics analysis in predicting the meningioma grade using multiparametric MRI. Eur J Radiol. 2020, 131:109251. 10.1016/j.ejrad.2020.109251

8. Apra C, Peyre M, Kalamarides M: Current treatment options for meningioma . Expert Rev Neurother. 2018, 18:241-9. 10.1080/14737175.2018.1429920

9. Bulleid LS, James Z, Lammie A, Hayhurst C, Leach PA: The effect of the revised WHO classification on the incidence of grade II meningioma. Br J Neurosurg. 2020, 34:584-6. 10.1080/02688697.2019.1639616

10. Perry A, Stafford SL, Scheithauer BW, Suman VJ, Lohse CM: Meningioma grading: an analysis of histologic parameters. Am J Surg Pathol. 1997, 21:1455-65. 10.1097/00000478-199712000-00008

11. Zeidman LA, Ankenbrandt WJ, Du H, Paleologos N, Vick NA: Growth rate of non-operated meningiomas. J Neurol. 2008, 255:891-5. 10.1007/s00415-008-0801-2

12. Behbahani M, Skeie GO, Eide GE, Hausken A, Lund-Johansen M, Skeie BS: A prospective study of the natura history of incidental meningioma-Hold your horses!. Neurooncol Pract. 2019, 6:438-50. 10.1093/nop/npz011

13. R: a language and environment for statistical computing . (2020). Accessed: January 20, 2022: https://www.R-project.org/.

14. Zhang S, Chiang GC, Knapp JM, et al.: Grading meningiomas utilizing multiparametric MRI with inclusion of susceptibility weighted imaging and quantitative susceptibility mapping. J Neuroradiol. 2020, 47:272-7. 10.1016/j.neurad.2019.05.002

15. Gurkanlar D, Er U, Sanli M, Ozkan M, Sekerci Z: Peritumoral brain edema in intracranial meningiomas . J Clin Neurosci. 2005, 12:750-3. 10.1016/j.jocn.2004.09.029

16. Salah F, Tabbarah A, ALrab Y N, et al.: Can CT and MRI features differentiate benign from malignant meningiomas?. Clin Radiol. 2019, 74:898.e15-23. 10.1016/j.crad.2019.07.020

17. Varlotto J, Flickinger J, Pavelic MT, et al.: Distinguishing grade I meningioma from higher grade meningiomas without biopsy. Oncotarget. 2015, 6:38421-8. 10.18632/oncotarget.5376

18. Amano T, Nakamizo A, Michiwaki Y, Matsuo S, Fujioka Y, Nagata S: Surgical outcome in elderly patients with intracranial meningioma. J Clin Neurosci. 2018, 56:63-6. 10.1016/j.jocn.2018.07.009

19. Islim AI, Mohan M, Moon RD, Srikandarajah N, Mills SJ, Brodbelt AR, Jenkinson MD: Incidental intracranial meningiomas: a systematic review and meta-analysis of prognostic factors and outcomes. J Neurooncol. 2019, 142:211-21. 10.1007/s11060-019-03104-3

20. Jääskeläinen J, Haltia M, Laasonen E, Wahlström T, Valtonen S: The growth rate of intracranial meningiomas and its relation to histology. An analysis of 43 patients. Surg Neurol. 1985, 24:165-72. 10.1016/0090-3019(85)90180-6

21. Nakamura M, Roser F, Michel J, Jacobs C, Samii M: Volumetric analysis of the growth rate of incompletely resected intracranial meningiomas. Zentralbl Neurochir. 2005, 66:17-23. 10.1055/s-2004-836225

22. Nakasu S, Fukami T, Nakajima M, Watanabe K, Ichikawa M, Matsuda M: Growth pattern changes of meningiomas: long-term analysis. Neurosurgery. 2005, 56:946-55. 